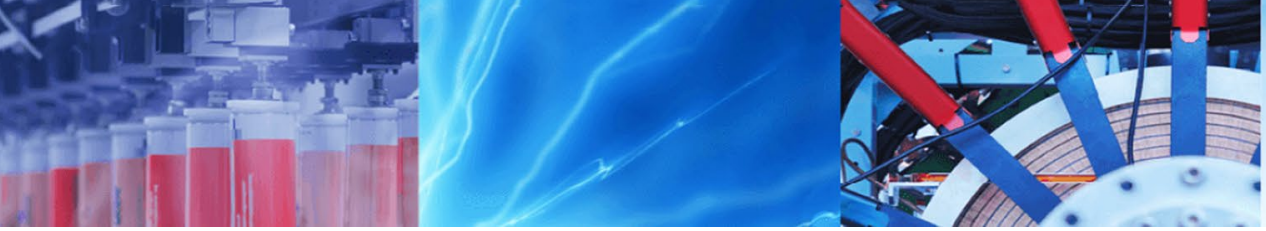

Research Article

\title{
Leapfrogging dynamics of interacting solitons in weakly coupled nonlinear transmission lines
}

\author{
Nkongho Achere Akem ${ }^{1,2} \cdot$ L. Akong Ngate ${ }^{1}$ Alain M. Dikandé ${ }^{1}$ (D) B. Z. Essimbi ${ }^{2}$
}

(c) Springer Nature Switzerland AG 2019

\begin{abstract}
The dynamics of two electrical pulses forming a boundstate, propagating along two nonlinear transmission lines weakly coupled by linear capacitors shunted with linear resistances, is considered from both analytical and numerical standpoints. The study rests on an analysis of time series of the amplitudes and phases of the two interacting electrical pulses, within the framework of the variational theory based on exact one-soliton solution to the Korteweg-de Vries equation. In the regime where the two pulses propagate at nearly equal velocities, their relative amplitude/phase evolutions can result in periodic quasi-harmonic oscillations so-called leapfrogging motion. In this specific regime of motion, it is found that besides the expected damping effect on the soliton amplitudes, the resistance can also sustain their leapfrogging motion. Analytical expression of the leapfrogging frequency is derived, providing a better understanding of the competing effects of the coupling capacitor and the resistive shunt on the leapfrogging motion. Leapfrogging motions of co-propagating pulses in electrical networks can be very useful in high-intensity signal transmissions involving least energy cost for the propagating signals.
\end{abstract}

Keywords Coupled nonlinear transmission lines · Soliton pairs · Leapfrogging $\cdot \mathrm{KdV}$ equations $\cdot$ Numerical simulations

\section{Introduction}

Nonlinear transmission lines (NLTLs) are dispersive media in which electric signals propagate in form of well-localized pulses [1-13], usually referred to as electrical solitons. Owing to their robustness that is their ability to cover long distances (thousands of kilometers) without change in profiles, electrical solitons have been actively investigated over the past fifty years starting from the pioneer model of Hirota and Suzuki [1, 2, 14-23]. In the microwave domain in particular NLTLs have recently been shown [21] to represent the ideal source of stable high-intensity sharp pulses.

Typically a NLTL is constructed by periodically loading a linear transmission line with reverse-biased semiconductor diodes (such as Schottky varactors), or by arranging inductors and varactors in a one-dimensional (1D) lattice. In such structures nonlinearity originates from the varactors whose capacitance is designed to change with applied voltage, while line dispersion stems from the structural periodicity of the loaded elementary circuits composing the ladder transmission line. When nonlinearity and dispersion are balanced, electrical currents and all related physical parameters (such as the voltages) acquire solitonic shape profiles and thus long-lived high-intensity electric signals are generated [15]. In addition to maintaining their shapes during propagation, solitons possess other important properties $[15,24]$. Namely they can survive collisions with other solitons or solitary waves, and in electrical transmission lines specifically it was recently established [25-27] that they can retain their profiles upon scatterings with localized as well as distributed structural defects (i.e. impurities) on the lines. Current applications

$\triangle$ Alain M. Dikandé, amdikande@gmail.com | 'Laboratory of Research on Advanced Materials and Nonlinear Science (LaRAMaNS), Department of Physics, Faculty of Sciences, University of Buea, PO Box 63, Buea, Cameroon. ${ }^{2}$ Present Address: Laboratory of Electronics and Electrical Systems, Department of Physics, Faculty of Science, University of Yaoundé I, P.O. Box 812, Yaoundé, Cameroon. 
involving NLTLs are numerous, under large signal conditions they can serve as impulse compressors and frequency multipliers [21], they are utilized for transportation of electrical energy through overhead high voltage cables, they serve as metal strips on printed circuit boards to carry digital data or control signals, they are utilized as coupled microstrips in microwave filters, as ribbon cables to connect electronic systems and so on. Resistive NLTLs are ideal devices for the study of short-pulse amplitude control [20, 28] and reshaping [21].

Nonlinear electrical networks, composed of at least two coupled nonlinear transmission lines, have been considered in the studies of simultaneous propagation of electrical soliton packets obeying coupled Korteweg-de Vries (KdV) equations [18, 29-32]. Theoretical as well as experimental results, in the specific context of two coupled NLTLs, have revealed a rather complex dynamics of the interacting pulses as they propagate together. In particular when two electrical pulses propagate along two separate but coupled transmission lines, the strength of their interaction mediated by the coupling will usually depend on their speeds one relative to the other. It was observed experimentally [33] that when the difference in velocities of the two pulses is very small, their interaction is optimized thus favoring a bound state in which electrical energy will be alternately transferred from a leading soliton to a trailing soliton. In this process, when one of the two solitons in the bound state is at its maximum amplitude, it leads the pair motion while the trailing soliton is at its minimum amplitude. As the energy leaves the leading soliton to the trailing soliton, the amplitude of the leading soliton dies down while the amplitude of the trailing soliton grows. Given that the velocity of the KdV soliton increases with amplitude [24], the trailing soliton is expected to speed up and to eventually overtake the leading soliton such that the direction of energy transfer is reversed. Under certain conditions this overtaking will occur repeatedly, giving rise to relative oscillations in amplitudes and phases in which the two solitons continuously leap over each other periodically. Such motion, called "leapfrogging", was discussed in ref. [33] in the context of capacitively coupled nonlinear transmission lines. Actually the leapfrogging of solitons is a quite common phenomenon in nature, it has been widely discussed in the past particularly in fluid dynamics [34-40]. The leapfrogging of electrical solitons may offer the possibility to quasi-resonantly transfer energy between coupled ladder lines in distributed electric networks, such process can be of great interest for space and time multiplexed ultrafast electronics.

In this work we are interested in the leapfrogging dynamics of a bound soliton pair propagating in two NLTLs, weakly coupled by a capacitive component as considered in Ref. [33] but shunted with a linear resistance. Our main objective is to examine the influence of the resistive component on relative amplitude and phase oscillations of the two interacting solitons. As a matter of fact, if the damping of soliton amplitudes due to resistive components along NLTLs is a well established fact, we are anticipating a control role of the resistance in the leapfrogging dynamics of the two interacting electrical pulses.

\section{Model and coupled KdV equations}

The model of two coupled nonlinear transmission lines we are interested in is depicted in Fig. 1. The two lines consist of periodically loaded identical LC elementary circuits, where sections of elementary circuits in line 1 and line 2 are connected by interfacial linear capacitors $C_{m}$ shunted by linear resistors $R_{m}$. Dispersion in the system is achieved by the discrete nature of the lines, while nonlinearity is achieved by making use of the conductive nonlinearity of capacitive diodes (i.e. varactors) [41]. In our study we assume the case when the varactors in the two lines are connected with opposite polarities.
Fig. 1 Equivalent representation of two nonlinear LC transmission lines, coupled by a capacitor $C_{m}$ with a resistance $R_{m}$ in its shunt branch

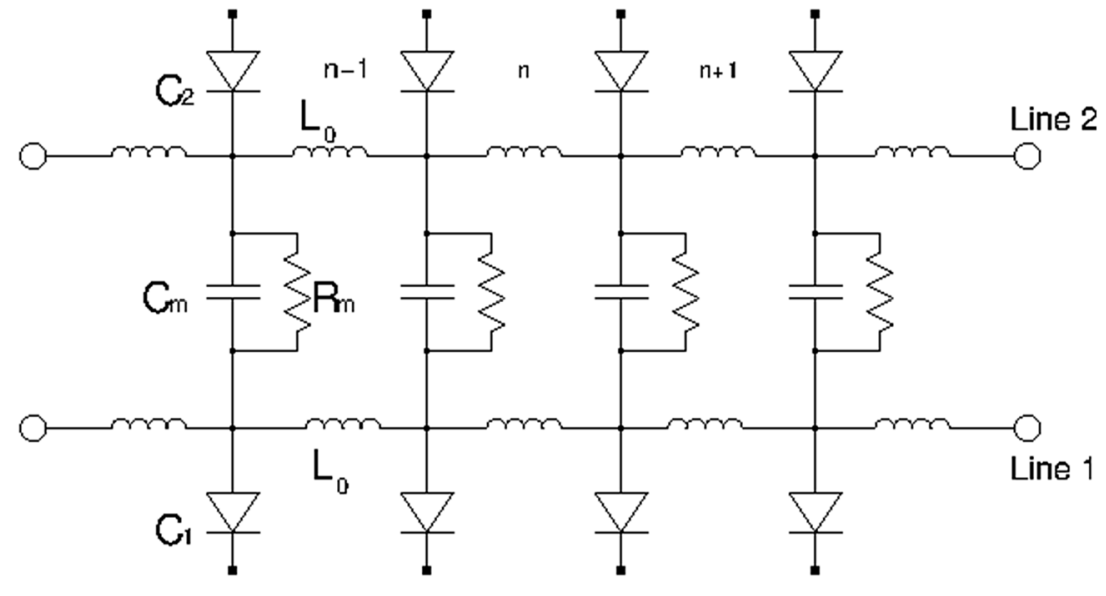


Let $V_{n}$ and $I_{n}$ denote the voltage and current respectively, at the $n$th section of line 1 , and $W_{n}$ and $J_{n}$ the voltage and current respectively at the $n$th section of line 2 . The bias voltages in lines 1 and 2 are $-V_{b}$ and $V_{b}$ respectively, reflecting the opposite polarities of the varactor diodes on the two lines. For these varactor diodes we assume the following capacitance-voltage characteristics [42]:

$$
C_{1}(x)=C_{0}\left(1-\frac{x}{V_{J}}\right)^{-m}, \quad C_{2}(x)=C_{0}\left(1+\frac{x}{V_{J}}\right)^{-m}
$$

where $C_{0}$ is the zero-bias junction capacitance, $V_{J}$ is the junction potential and $m$ (a real number) is a grading index coefficient determined by the doping profile of the varactors $[41,42]$. Note that except for the opposite polarities of the varactor diodes the lines are symmetrical, this symmetry turns out to be fundamental for it favors electrical pulses of almost equal velocities to travel on the two lines as a bound state.

Applying Kirchhoff's voltage law on the $n^{\text {th }}$ cell of lines 1 and 2, we obtain:

$L_{0} \frac{d I_{n}}{d t}=V_{n-1}-V_{n}, \quad L_{0} \frac{d J_{n}}{d t}=W_{n-1}-W_{n}$.

Kirchhoff's current law on the $n^{\text {th }}$ cell of lines 1 and 2 results in the two coupled transmission line equations:

$$
\begin{aligned}
& C_{1}\left(V_{n}\right) \frac{d^{2} V_{n}}{d t^{2}}+C_{m} \frac{d^{2}}{d t^{2}}\left(V_{n}-W_{n}\right)+\frac{1}{R_{m}} \frac{d}{d t}\left(V_{n}-W_{n}\right) \\
& =L_{0}^{-1}\left(V_{n+1}-2 V_{n}+V_{n-1}\right), \\
& C_{2}\left(W_{n}\right) \frac{d^{2} W_{n}}{d t^{2}}+C_{m} \frac{d^{2}}{d t^{2}}\left(W_{n}-V_{n}\right)+\frac{1}{R_{m}} \frac{d}{d t}\left(W_{n}-V_{n}\right) \\
& =L_{0}^{-1}\left(W_{n+1}-2 W_{n}+W_{n-1}\right) .
\end{aligned}
$$

In the long-wavelength limit, we can approximate the discrete position $n$ by a continuous variable $x=n h$ where $h$ is the size of an elementary circuit. In this continuous regime the discrete voltages transform as $V_{n}(t) \rightarrow V(x, t)$ and $W_{n}(t) \longrightarrow W(x, t)$, such that we can carry out a continuum-limit expansion in powers of $h$, for the two quantities $V_{n \pm 1}(t)=V(x \pm h, t)$ and $W_{n \pm 1}(t)=W(x \pm h, t)$. To the fourth order in $h$ the expansion yields:

$$
V_{n \pm 1}(t)=V(x, t) \pm h \frac{\partial V}{\partial x}+\frac{h^{2}}{2 !} \frac{\partial^{2} V}{\partial x^{2}} \pm \frac{h^{3}}{3 !} \frac{\partial^{3} V}{\partial x^{3}}+\frac{h^{4}}{4 !} \frac{\partial^{4} V}{\partial x^{4}}+0\left(h^{5}\right),
$$

$W_{n \pm 1}(t)=W(x, t) \pm h \frac{\partial W}{\partial x}+\frac{h^{2}}{2 !} \frac{\partial^{2} W}{\partial x^{2}} \pm \frac{h^{3}}{3 !} \frac{\partial^{3} W}{\partial x^{3}}+\frac{h^{4}}{4 !} \frac{\partial^{4} W}{\partial x^{4}}+0\left(h^{5}\right)$.

Setting $h \equiv 1$ for simplicity, Eqs. (3) and (4) become:

$$
\begin{aligned}
& C_{1}(V) \frac{\partial^{2} V}{\partial t^{2}}+C_{m} \frac{\partial^{2}}{\partial t^{2}}(V-W)+\frac{1}{R_{m}} \frac{\partial}{\partial t}(V-W) \\
& =L_{0}^{-1}\left(\frac{\partial^{2} V}{\partial x^{2}}+\frac{1}{12} \frac{\partial^{4} V}{\partial x^{4}}\right), \\
& C_{2}(W) \frac{\partial^{2} W}{\partial t^{2}}+C_{m} \frac{\partial^{2}}{\partial t^{2}}(W-V)+\frac{1}{R_{m}} \frac{\partial}{\partial t}(W-V) \\
& =L_{0}^{-1}\left(\frac{\partial^{2} W}{\partial x^{2}}+\frac{1}{12} \frac{\partial^{4} W}{\partial x^{4}}\right),
\end{aligned}
$$

where $C_{1}(V)$ and $C_{2}(W)$ are defined in (1).

\section{Adiabatic equations}

As we are interested in electric signals with localized pulse shapes, we shall follow the reductive perturbation theory $[35,43]$ to obtain the equations governing spatiotemporal evolutions of such localized electric signals. In this respect, we introduce a small parameter $\varepsilon$ and define the new variables:

$s=\varepsilon^{1 / 2}\left(x-v_{0} t\right), \quad \tau=\varepsilon^{3 / 2} t$,

with $v_{0}=1 / \sqrt{L_{0} C_{b}}$. Next, express the voltages $V(s, \tau)$ and $W(s, \tau)$ as series in powers of $\varepsilon$ i.e.:

$V(s, \tau)=-V_{b}+\sum_{i=1}^{\infty} \varepsilon^{i} V_{i}(s, \tau)$,

$W(s, \tau)=V_{b}+\sum_{i=1}^{\infty} \varepsilon^{i} W_{i}(s, \tau)$

Consistently with the weak-coupling assumption, the coupling capacitance $C_{m}$ and coupling resistance $R_{m}$ will be taken of the order $\varepsilon$. In agreement with this assumption, we can rewrite the two coupling parameters as:

$C_{m}=\varepsilon C_{m^{\prime}}^{\prime}$

$R_{m}=\varepsilon^{-3 / 2} R_{m}^{\prime}$.

Substituting Eqs. (9), (10), (11), (12) and (13) into Eqs. (7) and (8), and keeping only terms of order $\varepsilon^{3}$ for which nonlinearity balances the dispersion, we obtain the following two coupled KdV equations at the third order in $\varepsilon$ :

$v_{\tau^{\prime}}^{\prime}-6 v^{\prime} v_{s^{\prime}}^{\prime}+v_{s^{\prime} s^{\prime} s^{\prime}}^{\prime}=\eta\left(v^{\prime}+w^{\prime}\right)_{s^{\prime}}-\eta_{1}\left(w^{\prime}+v^{\prime}\right)$,

$w_{\tau^{\prime}}^{\prime}-6 w^{\prime} w_{s^{\prime}}^{\prime}+w_{s^{\prime} s^{\prime} s^{\prime}}^{\prime}=\eta\left(w^{\prime}+v^{\prime}\right)_{s^{\prime}}-\eta_{1}\left(v^{\prime}+w^{\prime}\right)$,

where we define: 


$$
\begin{aligned}
\tau^{\prime} & =\frac{\tau}{\beta_{1}}, \quad s^{\prime}=\sqrt[3]{12 C_{0} L_{0}} S \\
v^{\prime} & =6 \beta_{2} \sqrt[3]{12 C_{0} L_{0}} v_{3}, \quad w^{\prime}=-6 \beta_{2} \sqrt[3]{12 C_{0} L_{0}} w_{3} \\
\eta & =\frac{C_{m}^{\prime} v_{0}^{2} \sqrt[3]{12 C_{0} L_{0}}}{C_{0}}, \quad \eta_{1}=\frac{v_{0} r_{m}}{C_{0}},
\end{aligned}
$$

with $\beta_{1}=2 v_{0}-4 \beta_{2} v_{0} V_{b}, \beta_{2}=\frac{m}{v_{j}}$, and for convenience we have set $r_{m}=R_{m}^{\prime}$. Contributions from terms of order $\varepsilon$ give $v_{1}=0$ and $w_{1}=0$, while contributions of order $\varepsilon^{2}$ (i.e. $v_{2}$ and $w_{2}$ ) are determined by the following equations:

$0=C_{0} v_{0}^{2} \frac{\partial^{2} v_{2}}{\partial s^{2}}-2 C_{0} v_{0}^{2} \alpha_{1} v_{b} \frac{\partial^{2} v_{2}}{\partial s^{2}}+\frac{1}{12 L_{0}} \frac{\partial^{2} v_{2}}{\partial s^{2}}$

$0=C_{0} v_{0}^{2} \frac{\partial^{2} w_{2}}{\partial s^{2}}-2 C_{0} v_{0}^{2} \alpha_{1} v_{b} \frac{\partial^{2} w_{2}}{\partial s^{2}}+\frac{1}{12 L_{0}} \frac{\partial^{2} w_{2}}{\partial s^{2}}$

suggesting that $v_{2}$ and $w_{2}$ are constants.

The two coupled Eqs. (14) and (15), that provide shape profiles of the nonlinear components of the voltage fields experessed as in series as (10), (11), are not tractable analytically because of the coupling terms in their right-hand side. However, if we assume the two coupling components to be very small, they can readily be treated as perturbations and therefore we can rewrite the set (14) and (15) formally with their right-hand side grouped to give a single perturbation function:

${ }_{\epsilon} P\left(v^{\prime}, w^{\prime}\right)=\eta\left(v^{\prime}+w^{\prime}\right)_{s^{\prime}}-\eta_{1}\left(w^{\prime}+v^{\prime}\right)$.

In the absence of perturbation, i.e. when $\epsilon P\left(v^{\prime}, w^{\prime}\right)=0$, Eqs. (14) and (15) are two independent KdV equations whose exact single-pulse soliton solutions are [35]:

$v^{\prime}=-2 \kappa_{1}^{2} \operatorname{sech}^{2}\left(z_{1}\right), \quad w^{\prime}=-2 \kappa_{2}^{2} \operatorname{sech}^{2}\left(z_{2}\right)$,

where the arguments $z_{1}=\kappa_{1}\left(s^{\prime}-\zeta_{1}\right)$ and $z_{2}=\kappa_{2}\left(s^{\prime}-\zeta_{2}\right)$ connect the pulse amplitudes $\kappa_{1,2}$ to their phases $\zeta_{1,2}$ via:

$\zeta_{1}=4 \kappa_{1}^{3} \tau^{\prime}, \quad \zeta_{2}=4 \kappa_{2}^{3} \tau^{\prime}$.

It is worthwhile stressing that (20) are just parts of the general solutions to the two line equations, however they are most relevant in that they provide the soliton components in the series representation of these solutions. Hence we shall be mainly interested in the dynamics of these soliton components in the presence of the perturbation $\epsilon P\left(v^{\prime}, w^{\prime}\right)=0$. To determine the temporal evolution of characteristic parameters $\kappa_{i}$ and $\zeta_{i}$ of the two pulse solitons for small $\epsilon P\left(v^{\prime}, w^{\prime}\right)$, we use the adiabatic perturbation theory [35] which enables us obtain the following set of first-order ordinary differential equations:

$\frac{d \kappa_{i}}{d \tau^{\prime}}=-\frac{\eta}{4 \kappa_{i}} \int_{-\infty}^{\infty} \epsilon P\left(v^{\prime}, w^{\prime}\right) \operatorname{sech}^{2}\left(z_{i}\right) d z_{i}$,

$$
\begin{aligned}
\frac{d \zeta_{i}}{d \tau^{\prime}}= & 4 \kappa_{i}^{2}-\frac{\eta}{4 \kappa_{i}^{3}} \int_{-\infty}^{\infty} \epsilon P\left(v^{\prime}, w^{\prime}\right) \operatorname{sech}^{2}\left(z_{i}\right) \\
& \times\left(z_{i}+\frac{1}{2} \sinh \left(2 z_{i}\right)\right) d z_{i}
\end{aligned}
$$

with $i=1$, 2 . Note that the interaction between the two solitons will be optimal when their velocities coincide, i.e. $\dot{\zeta}_{1}=\dot{\zeta}_{2}$. In terms of (20) this can also be formulated in terms of the equality of their amplitudes, i.e.:

$\kappa_{1}=\kappa_{2}$.

Let us consider a very small deviation from this optimal state of interaction, due to the soliton velocities not being exactly equal but remaining nevertheless always very close. This can be described by small fluctuations in amplitudes of the two pulses from their unperturbed values $\kappa_{1}^{0}=\kappa_{2}^{0}=\kappa$. It follows that since the two lines are identical and symmetric, we can write:

$\kappa_{1,2}=\kappa+\lambda_{1,2}$

where $\kappa$ is constant and the variables $\lambda_{1}$ and $\lambda_{2}$ are very small compared to $\kappa$. Similarly the phase difference $\Delta \zeta \equiv \zeta_{1}-\zeta_{2}$ can be assumed so small that we have:

$z_{2} \simeq z_{1}+\kappa \Delta \zeta$.

Substituting Eqs. (20), (25) and (26) in (22) and (23) and integrating, we obtain:

$$
\begin{aligned}
\frac{d \lambda_{1}}{d \tau^{\prime}}= & -\frac{\eta \kappa_{2}^{3}}{15 \kappa_{1}} \operatorname{sech}^{2}(y)\left[8 \tanh (y)+8 \tanh ^{3}(y)\right] \\
& -\frac{2 \eta_{1} \kappa_{2}^{2}}{3 \kappa_{1}} \operatorname{sech}^{2}(y)-\frac{2 \eta_{1} \kappa_{1}}{3}
\end{aligned}
$$

$$
\begin{aligned}
\frac{d \lambda_{2}}{d \tau^{\prime}}= & \frac{\eta \kappa_{1}^{3}}{15 \kappa_{2}} \operatorname{sech}^{2}(y)\left[8 \tanh (y)+8 \tanh ^{3}(y)\right] \\
& -\frac{2 \eta_{1} \kappa_{1}^{2}}{3 \kappa_{2}} \operatorname{sech}^{2}(y)-\frac{2 \eta_{1} \kappa_{2}}{3}
\end{aligned}
$$

$$
\begin{aligned}
\frac{d \zeta_{1}}{d \tau^{\prime}}= & 4 \kappa_{1}^{2}-\eta-\frac{\eta \kappa_{2}^{3}}{3 \kappa_{1}^{3}} \operatorname{sech}^{2}(y)\left[3-\frac{17}{3} \tanh ^{2}(y)\right] \\
& +\frac{\eta_{1} \kappa_{2}^{2}}{\kappa_{1}^{3}} \operatorname{sech}^{2}(y) \tanh (y)
\end{aligned}
$$

$$
\begin{aligned}
\frac{d \zeta_{2}}{d \tau^{\prime}} & =4 \kappa_{2}^{2}-\eta-\frac{\eta \kappa_{1}^{3}}{3 \kappa_{2}^{3}} \operatorname{sech}^{2}(y)\left[3-\frac{17}{3} \tanh ^{2}(y)\right] \\
& -\frac{\eta_{1} \kappa_{1}^{2}}{\kappa_{2}^{3}} \operatorname{sech}^{2}(y) \tanh (y)
\end{aligned}
$$

\section{SN Applied Sciences}


where we have set $y=\kappa \Delta \zeta$. Eqs. (27)-(30), that describe the motion of the two weakly interacting electrical solitons in the specific context when their velocities are very close, are solved numerically using a sixth-order RungeKutta scheme [44] with fixed step. Numerical results are discussed in the next section.

\section{Numerical results}

To investigate the leapfrogging dynamics of the two electrical solitons we carried out numerical simulations of the four coupled ordinary differential equations Eqs. (27)-(30). Since it is also relevant to see the effects the coupling resistance would have on amplitudes of the individual solitons, in Fig. $2 a$, b we started with plots of the time series of $\lambda_{1}$ and $\lambda_{2}$, for a fixed value of the capacitive coupling $\eta(\eta=0.025)$, for different values of the resistive coupling parameter i.e. $\eta_{1}=0.001,0.005$ and 0.009 . One sees that time series of $\lambda_{1}$ and $\lambda_{2}$ are periodic oscillations, with an exponential decrease of their maxima. It is quite
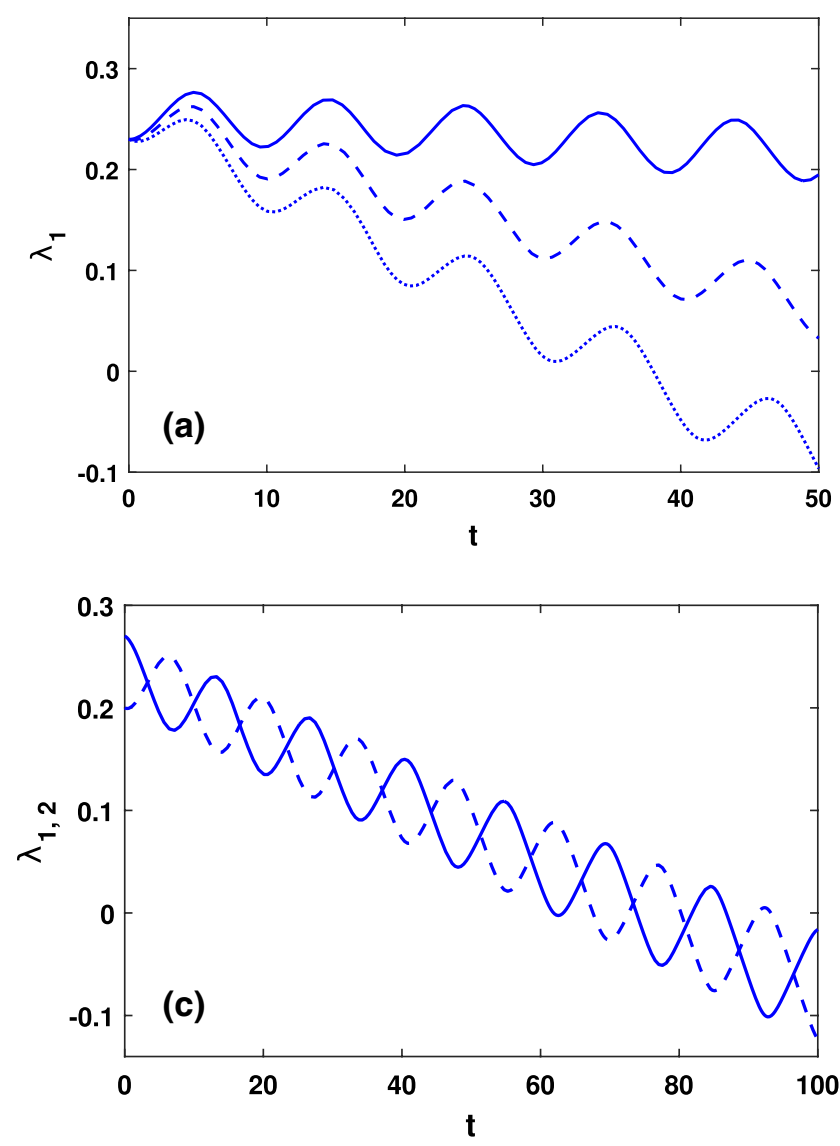

Fig. 2 (Color online) Top graphs: Temporal evolutions of $\lambda_{1}(\mathbf{a})$ and $\lambda_{2}(\mathbf{b})$, for $\eta=0.23$ and $\kappa=0.6$. The solid, dashed and dotted curves correspond respectively to $\eta_{1}=0.001,0.005$ and 0.009 . Bottom remarkable that the exponential damping is more and more pronounced as $\eta_{1}$ increases.

To highlight the anti-phase oscillations of the two amplitudes, we plot $\lambda_{1}$ and $\lambda_{2}$ on the same graph for large values of $\eta_{1}\left(\eta_{1}=0.005\right.$ and $\left.\eta_{1}=0.009\right)$ keeping $\eta=0.025$ and $\kappa=0.5$. The leapfrogging motions observed in Fig. 2c, $\mathrm{d}$, occur more exactly when initial values of $\lambda_{1}$ and $\lambda_{2}$ used in the simulations are very close. Figure 3 illustrates the absence of leapfrogging, when the difference between initial values of the two parameters in the simulations is relatively large. In fact, the signature of leapfrogging is to be observed both in the oscillating phase difference $\Delta \zeta=\zeta_{1}-\zeta_{2}$, and in the oscillating amplitude difference $\Delta \lambda=\lambda_{1}-\lambda_{2}$ of the two solitons. Therefore, to gain a consistent picture of this signature in the two parameters, we solved numerically the amplitude and phase difference equations derived from the coupled set Eqs. (27)-(30), by substracting Eq. (29) from Eq. (30) on one hand and Eq. (27) from Eq. (28) on the other hand. Figure 4 suggests a dynamics of the soliton pair which we can summarize as follows: initially (i.e. at $t=0$ ) the amplitude difference of the two solitons is at some finite initial value, while
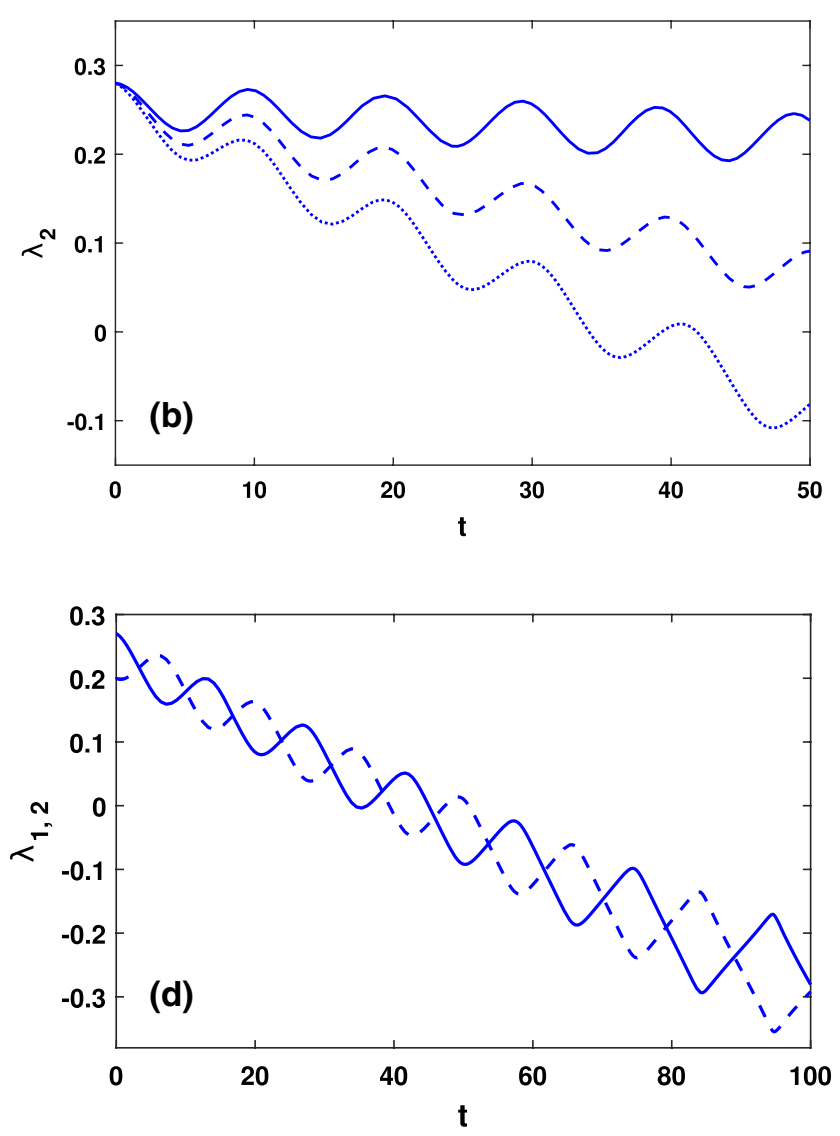

graphs: Leapfrogging dynamics of $\lambda_{1}$ (solid curve) and $\lambda_{2}$ (dashed curve), for $\eta_{1}=0.005$ (c) and $\eta_{1}=0.009$ (d) 


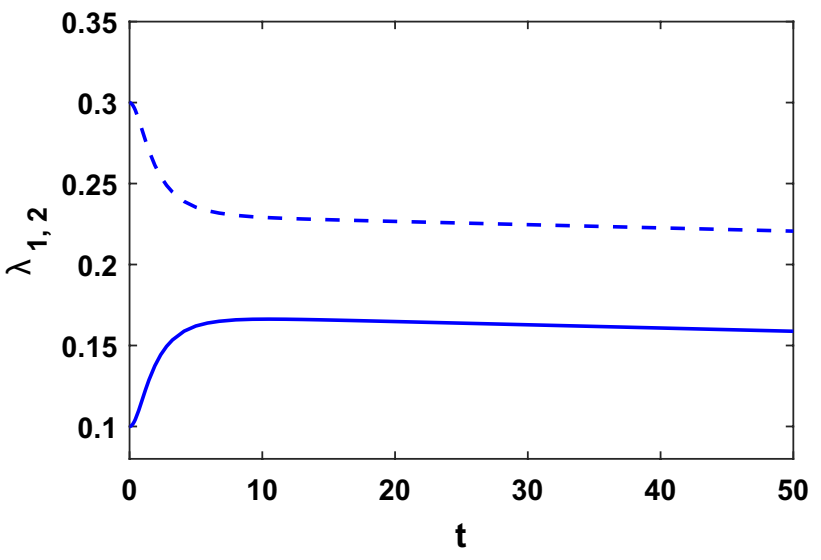

Fig. 3 (Color online) Suppression of leapfrogging for large initial values of $\lambda_{1}$ (i.e. 0.1 ) and $\lambda_{2}$ (i.e. 0.2 ), with $\eta=0.25, \eta_{1}=0.0005$ and $\kappa=0.5$

their phase difference is zero. As the two solitons propagate they exchange energy, this causes them to slowly approach each other. Their amplitude difference thus decreases gradually to zero at the time when the two electrical pulses coincide and have an equal velocity. After hopping pass each other, their amplitude and phase differences begin to rise again and the cycle continues, for as long as the leapfrogging motion goes on resulting in oscillating amplitude and phase differences.

To further emphasize the effects of the resistance on the leapfrogging dynamics of the bound solitons, we have plotted three sets of curves to illustrate the behaviour of the oscillating amplitude difference and phase difference, with increasing resistance (Fig. 5). Using fairly large values for the resistance, the curves clearly show that as the resistance increases the number of oscillations in $\Delta \lambda$ and $\Delta \zeta$ decreases until the leapfrogging motion stops. Since the oscillating evolutions of the amplitude and phase differences are here assumed to characterize the leapfrogging of the soliton pair, the decrease in the number of oscillations readily indicates a weakening and eventually a relaxation of the leapfrogging motion.

We can understand the origin of the harmonic oscillations observed in numerical solutions to the phase and amplitude difference equations, by remarking that the arguments of the hyperbolic functions in Eqs. (27)-(30) are proportional to $\Delta \zeta$. Thus for leapfrogging to occur in numerical simulations, this later parameter should remain very small which justifies our choice of very small initial values for the phase and amplitude differences. Analytically, small amplitude and phase differences imply linearizing Eqs. (27)-(30), which yields:

$\frac{d \lambda_{1}}{d \tau^{\prime}}=-\frac{8}{15} \eta \kappa^{2} y-\frac{4}{3} \eta_{1} k-\frac{4}{3} \eta_{1} \lambda_{2}$

$\frac{d \lambda_{2}}{d \tau^{\prime}}=\frac{8}{15} \eta \kappa^{2} y-\frac{4}{3} \eta_{1} k-\frac{4}{3} \eta_{1} \lambda_{1}$

$\frac{d \zeta_{1}}{d \tau^{\prime}}=4 \kappa_{1}^{2}-\eta-\frac{\eta \kappa_{2}^{3}}{4 \kappa_{1}^{3}}+\frac{\eta_{1} \kappa_{2}^{2} y}{\kappa_{1}^{3}}$

$\frac{d \zeta_{2}}{d \tau^{\prime}}=4 \kappa_{2}^{2}-\eta-\frac{\eta \kappa_{2}^{3}}{4 \kappa_{1}^{3}}-\frac{\eta_{1} \kappa_{1}^{2} y}{\kappa_{2}^{3}}$

Subtracting Eq. (34) from (33) and replacing in (31) and (28), we obtain:

$\frac{d^{2} \Delta \zeta}{d \tau^{\prime 2}}=\left(\frac{128}{15} \kappa^{4} \eta+\frac{96}{15} \kappa^{2} \eta^{2}-4 \eta_{1}^{2}\right) \Delta \zeta$

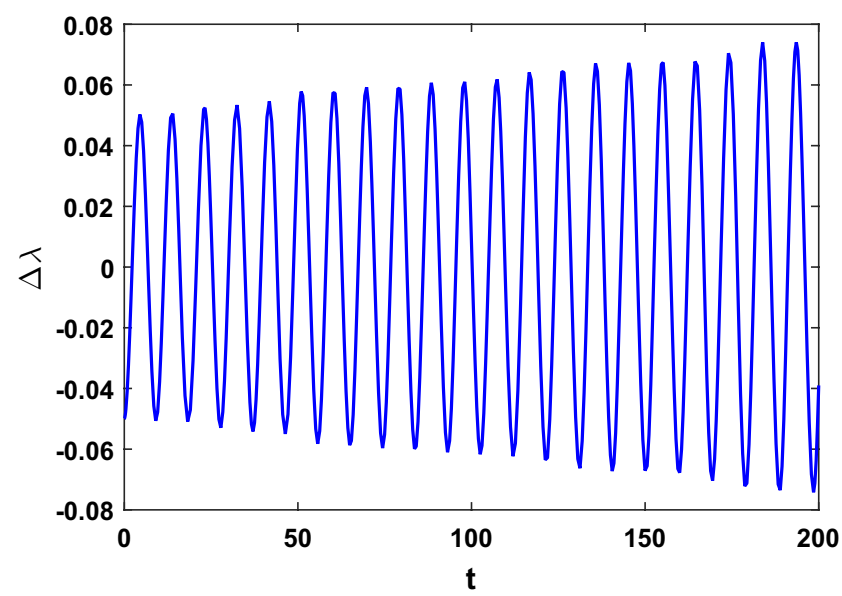

Fig. 4 (Color online) Temporal oscillations of the amplitude difference $\Delta \lambda$ and phase difference $\Delta \zeta$ are shown in the left and right graphs respectively, for $\eta=0.25, \eta_{1}=0.0005$, and $\kappa=0.63$ 
Fig. 5 (Color online) Oscillating amplitude and phase differences for increasing resistance. The top, middle and bottom set of curves are for $\eta_{1}=0.01$, 0.05 and 0.09 respectively. Here $\eta=0.25$ and $\kappa=0.63$. Leapfrogging ceases in the last set of curves due to very high value of resistance
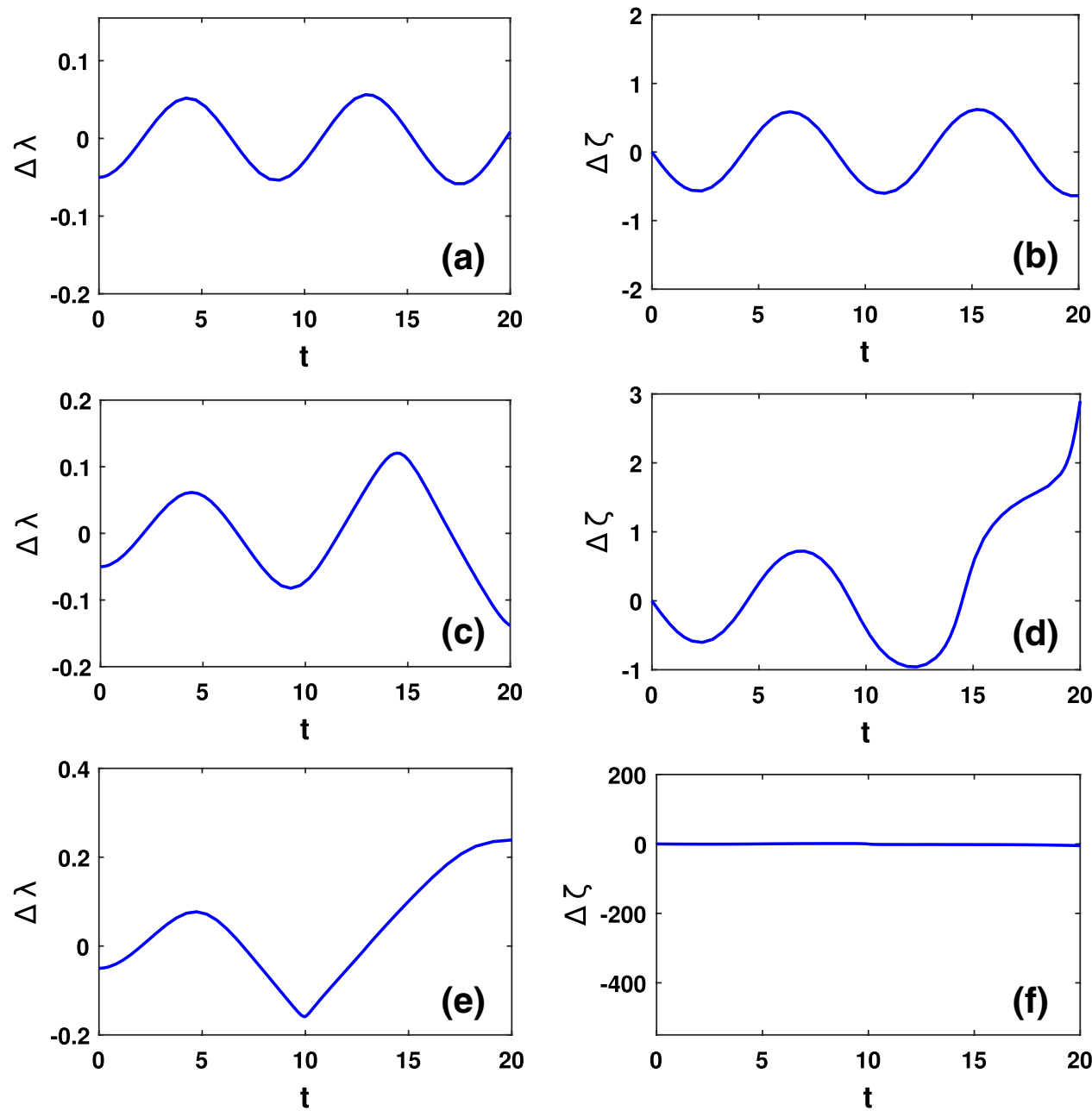

This is the equation of motion for an harmonic oscillator, the frequency $\omega_{\text {If }}$ of which is given by:

$\omega_{\text {If }}^{2}=\left(\frac{128}{15} \kappa^{4} \eta+\frac{96}{15} \kappa^{2} \eta^{2}-4 \eta_{1}^{2}\right)$.

Formula (36) shows that the leapfrogging frequency is decreased by the presence of the resistive element in the coupling branch. From the expression of $\omega_{\text {If }}$ it is also apparent that as $\eta_{1}$ increases the leapfrogging frequency decreases, vanishing at some finite characteristic value of the resistance. This is in agreement with the behaviour observed in Figs. 2a, b, c and 4a, b. In Fig. 6, we plot the leapfrogging frequency against the resistance for different values of $\kappa$. The frequency decreases to zero, indicating a decay of the leapfrogging motion as the resistance increases.

In the above analysis it emerged that a choice of small initial values for the amplitude and phase differences was necessary for the observation of leapfrogging in numerical simulations of the coupled set Eqs. (27)-(30). In Fig. 7, we plot the time series of $\lambda_{1}$ and $\lambda_{2}$ for relatively large values

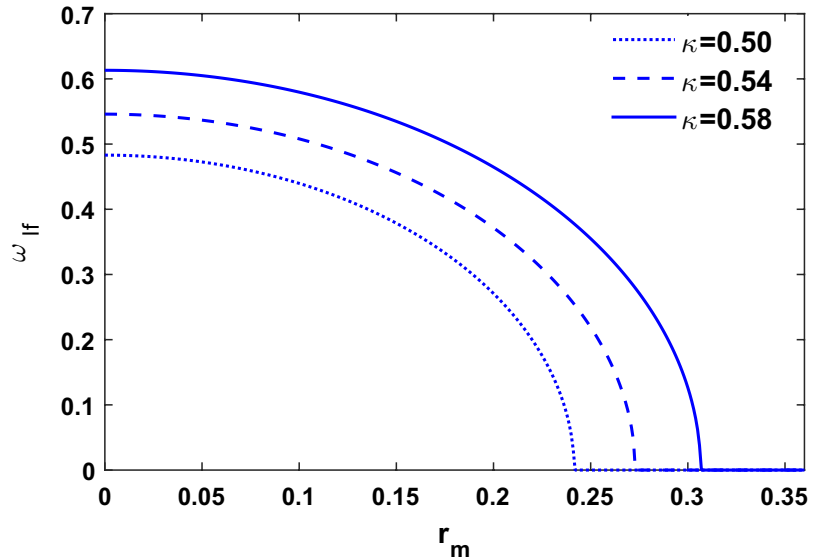

Fig. 6 (Color online) Influence of the resistance on the leapfrogging frequency for different values of $\kappa \cdot \eta=0.25$

of the initial phase and amplitude differences, considering different values of $\eta_{1}$ and $\kappa=0.6$. One sees that the time series of soliton characteristic parameters are now strongly 

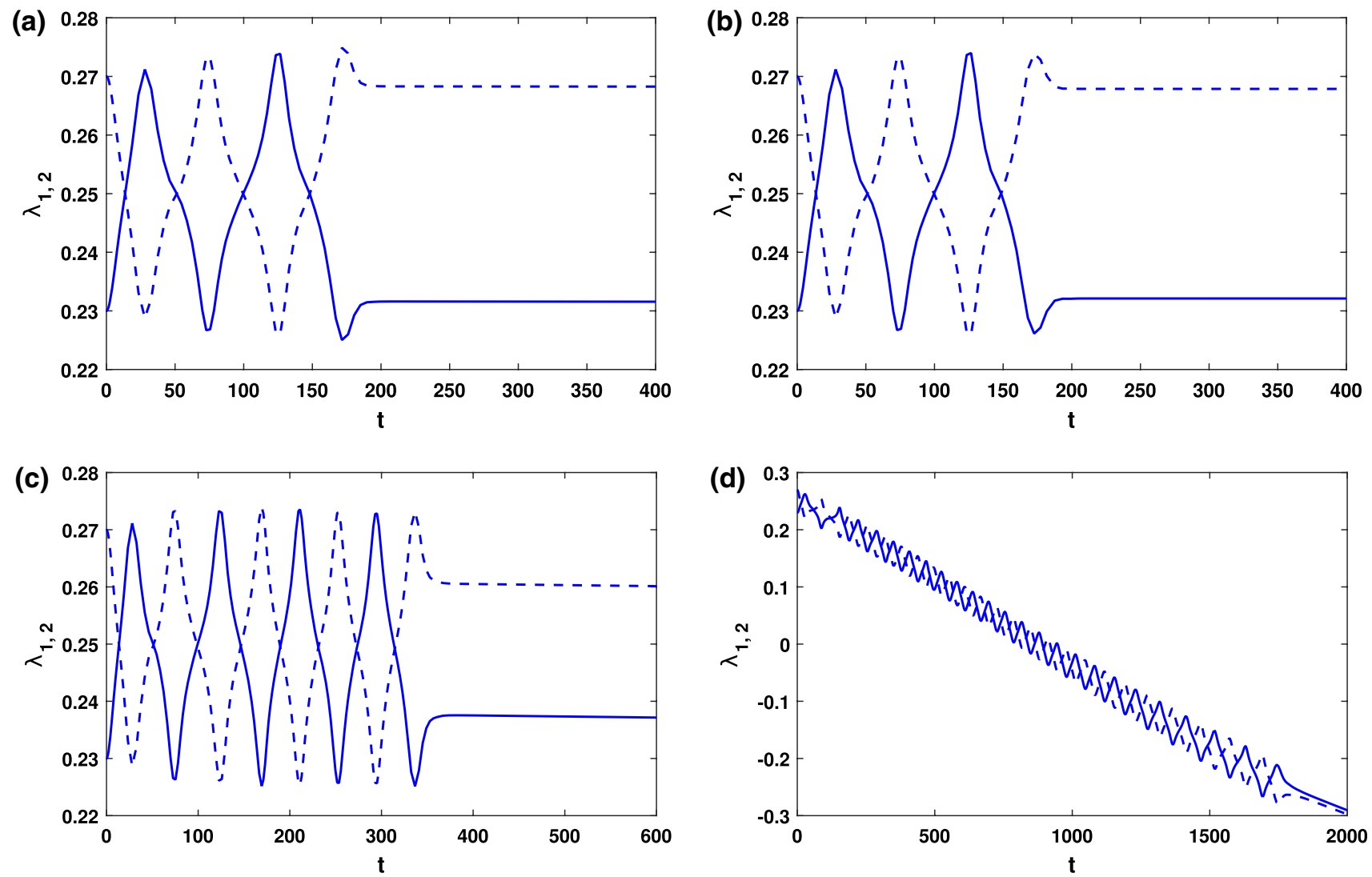

Fig. 7 (Color online) Anharmonic oscillations of $\lambda_{1}$ (solid curves) and $\lambda_{2}$ (dashed curves), for large initial values: $r_{m}=5 \times 10^{-10}(\mathbf{a})$, $r_{m}=5 \times 10^{-8}(\mathbf{b}), r_{m}=5 \times 10^{-6}(\mathbf{c})$ and $r_{m}=5 \times 10^{-4}(\mathbf{d}) . \kappa=0.6$

anharmonic, and more generally an enhancement of the anharmonicity by relatively large initial values will cause a relaxation of leapfrogging after a finite propagation time. Quite remarkably this later behaviour is valid irrespective of the value of the unperturbed amplitude $\kappa$, which indeed remains fix in time. To end, an explicit picture of the leapfrogging dynamics of the two solitons is summerized in the phase-space representation Fig. 8 , where $\lambda_{1}$ is plotted as a function of $\lambda_{2}$.

\section{Conclusion}

The leapfrogging of soliton pairs was proposed in the past to describe the particular motion of two weakly interacting solitons, characterized by their anti-phase oscillations and oppositely varying amplitudes [34-39, 45]. In fluid dynamics the leapfrogging of co-propagating KdV pulses have been widely discussed, and shown to provide an interesting configuration of the system dynamics in which solitons in the pair oscillate periodically one with respect to the other such as to mutually sustain their propagation at very small velocity [34]. This concept was recently extended to

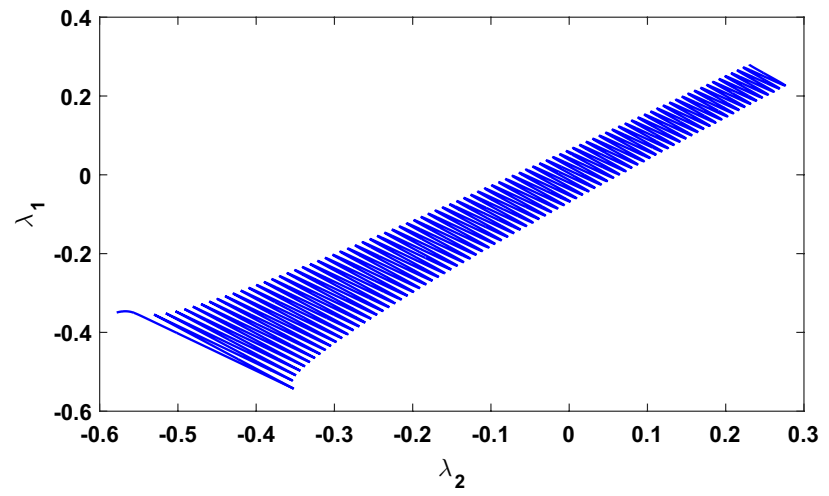

Fig. 8 (Color online) Phase-space representation of the leapfrogging dynamics. Here, $\eta_{1}=0.23, \eta=0.001$ and $\kappa=0.6$

nonlinear electrical transmission lines by Narahara [33], who considered two capacitively coupled nonlinear transmission lines and determined characteristic properties for the leapfrogging motion of electrical soliton pairs.

In the present work we examined the possible leapfrogging motion of a pair of solitons along two nonlinear LC transmission lines, weakly coupled by a linear 
capacitor shunted by a linear resistor. We established the leapfrogging motion by solving numerically the coupled variational equations associated with the two interacting solitons for very small initial values of the amplitude and phase differences, and obtained analytically that the resistive coupling component would cause a decrease in the leapfrogging frequency. Note that numerical curves also suggest a damping of amplitudes of the two solitons, also caused by the resistive coupling.

Although the results obtained in this work reveal a rich dynamics of the system, it would also be interesting to look at the competing effects of the coupling capacitance and resistance on the leapfrogging motion when the coupling parameters are not too small. Given that adiabatic considerations in this context cannot be applied, only a full numerical treatment of the discrete line equations would be consistent. This later study requires a specific context since several characteristic parameters are involved in the model and their contributions need to be well emphasized both quantitatively and qualitatively. This aspect is considered in a separate work.

\section{Compliance with ethical standards}

Conflict of interest The authors declare that they have no conflict of interest.

\section{References}

1. Hirota R, Suzuki K (1970) Studies on lattice solitons by using electrical networks. J Phys Soc Jpn 28:1366

2. Hirota R, Suzuki K (1973) Theoretical and experimental studies of lattice solitons in nonlinear lumped networks. Proc IEEE 61:1483

3. Kuusela T, Hietarinta J, Kokko K, Laiho R (1987) Soliton experiments in a nonlinear electrical transmission line. Eur J Phys 8:27

4. Kuusela T (1995) Soliton experiments in transmission lines. Chaos Solitons Fractals 5:2419

5. Mostafa SI (2009) Analytical study for the ability of nonlinear transmission lines to generate solitons. Chaos Solitons Fractals 39:2425

6. Dinkel JN, Setzer C, Rawal S, Lonngren KE (2001) Soliton propagation and interaction on a two-dimensional nonlinear transmission line. Chaos Solitons Fractals 12:91

7. Lin MM, Duan WS (2005) Wave packet propagating in an electrical transmission line. Chaos Solitons Fractals 24:191

8. Koon KT, Marquié P, Dinda PT (2014) Experimental observation of the generation of cutoff solitons in a discrete LC nonlinear electrical line. Phys Rev A 41:4534

9. Zayed EME, Alurrfi KAE (2015) A new Jacobi elliptic function expansion method for solving a nonlinear PDE describing the nonlinear low-pass electrical lines. Chaos Solitons Fractals 78:148

10. Kengne E (2004) Ginzburg-Landau system of complex modulation equations for a distributed nonlinear-dispersive transmission line. J Phys A 37:6053
11. Kengne E, Chui ST, Liu WM (2006) Modulational instability criteria for coupled nonlinear transmission lines with dispersive elements. Phys Rev E 74:0366141

12. Kengne E, Liu WM (2006) Exact solutions of the derivative nonlinear Schrödinger equation for a nonlinear transmission line. Phys Rev E 73:0266031

13. Kengne $E$, Lakhssassi $A$ (2015) Analytical studies of soliton pulses along two-dimensional coupled nonlinear transmission lines. Chaos Solitons Fractals 73:191

14. Jäger D (1982) Experiments on KdV Solitons. J Phys Soc Jpn $51: 1686$

15. Remoissenet M (1999) Waves called solitons: concepts and experiments. Springer, New York

16. Rodwell MJW et al (1994) Active and nonlinear wave propagation devices in ultrafast electronics and optoelectronics. Proc IEEE 82:1037

17. Essimbi BZ, Dikandé AM, Kofané TC, Zibi AA (1995) Asymmetric gap solitons in a non-linear LC transmission line. Phys Scr. 52:17

18. Essimbi BZ, Dikandé AM, Kofané TC, Zibi AA (1995) Gap solitons in nonlinear symmetric electric circuit. J Phys Soc Jpn 64:2777

19. Singer AC, Oppenheim AV, Wornell GW (1999) Detection and estimation of multiplexed soliton signals. IEEE Trans Signal Proc 47:2768

20. Narahara K (2010) Characterization of nonlinear transmission lines for short pulse amplification. J Infrared Millim Terahertz Waves 31:411

21. Afshari E, Hajimiri A (2005) Nonlinear transmission lines for pulse shaping in silicon. IEEE J Solid-State Circuits 40:744

22. Dikandé AM, Ga-Akeku B (2009) Localized short impulses in a nerve model with self-excitable membrane. Phys Rev $E$ 80:041904

23. Oriols X, Martin F (2001) Analytical solitons in nonlinear transmission lines loaded with heterostructure barrier varactors. J Appl Phys 90:2595

24. Drazin PG, Johnson RS (1989) Solitons: an introduction. Cambridge University Press, Cambridge

25. Sato M, Yasui S, Kimura M, Hikihara T, Sievers AJ (2007) Management of localized energy in discrete nonlinear transmission lines. Eur Phys Lett 80:30002

26. Tsuboi T, Toyama FM (1991) Computer experiments on solitons in a nonlinear transmission line. I. Formation of stable solitons. Phys Rev A 44:2686

27. Tsuboi T, Toyama FM (1991) Computer experiments on solitons in a nonlinear transmission line. II. Propagation of solitons in an impurity-doped line. Phys Rev A 44:2691

28. Gasch A, Berning T, Jäger D (1986) Generation and parametric amplification of solitons in a nonlinear resonator with a Korteweg-de Vries medium. Phys Rev A 34:4528

29. Yoshinaga T, Kakutani T (1980) Solitary and shock waves on a coupled transmission line. J Phys Soc Jpn 49:2072

30. Yoshinaga T, Kakutani $T$ (1987) Fast and slow mode solitons on a coupled transmission line. J Phys Soc Jpn 56:3447

31. Kofane TC, Zebaze M, Zibi AA (1990) Non-linear wave modulation on a coupled transmission line. J Phys D 23:764

32. Narahara K (2015) Asymmetrical solitary waves in coupled nonlinear transmission lines. Wave Motion 58:13

33. Narahara K (2015) Characterization of leapfrogging solitary waves in coupled nonlinear transmission lines. Nonlinear Dyn. 81:1805

34. Malomed BA (1987) Leapfrogging solitons in a system of coupled KdV equations. Wave Motion 9:401

35. Kivshar YS, Malomed BA (1989) Dynamics of solitons in nearly integrable systems. Rev Mod Phys 61:763

36. Liu AK, Pereira NR, Ko DRS (1982) Weakly interacting internal solitary waves in neighbouring pycnoclines. J Fluid Mech 122:187 
37. Weidman PD, Johnson M (1982) Experiments on leapfrogging internal solitary waves. J Fluid Mech 122:195

38. Gear JA, Grimshaw R (1984) Weak and strong interactions between internal solitary waves. Stud Appl Math 70:235

39. Nitsche M, Weidman PD, Grimshaw R, Ghrist M, Fornberg B (2010) Evolution of solitary waves in a two-pycnocline system. J Fluid Mech 642:235

40. Berger MS (1990) Mathematical structures of nonlinear science: an introduction. Kluwer, Dordrecht

41. Boylestad R, Nashelsky L (2013) Electronic devices and circuit theory $\left(7^{\text {th }}\right.$ edition. Prentice Hall, Englewood Cliffs

42. Jie H, Qian Z, Hao Y, Junrong D, Haiying Z (2014) Planar Schottky varactor diode and corresponding large signal model for millimeter-wave applications. J Semicond 35:0540061
43. Taniuti T, Wei CC (1968) Reductive perturbation method in nonlinear wave propagation. I. J Phys Soc Jpn 24:941

44. Luther HA (1968) An explicit sixth-order Runge-Kutta formula. Math Comput 22:434

45. Narahara K (2018) Modulation of pulse train using leapfrogging pulses developed in unbalanced coupled nonlinear transmission lines. Math Probl Eng, Article ID 2869731. https://doi. org/10.1155/2018/2869731

Publisher's Note Springer Nature remains neutral with regard to jurisdictional claims in published maps and institutional affiliations. 